\title{
In-silico identification of the binding mode of synthesized adamantyl derivatives inside cholinesterase enzymes
}

\author{
Amal AL-ABOUDI ${ }^{1, *}$, Raed A AL-QAWASMEH ${ }^{1}$, Alaa SHAHWAN ${ }^{1}$, Uzma MAHMOOD $^{2,4}$, Asaad KHALID ${ }^{3}$, Zaheer UL-HAQ ${ }^{4, *}$ \\ ${ }^{1}$ Department of Chemistry, The University of Jordan, Amman 11942, Jordan; ${ }^{2}$ Department of Bioinformatics, Aligarh Institute of \\ Technology, affiliated with Sir Syed University of Engineering \& Technology, Gulshan-e-lqbal, Block-5, Karachi 75300, Pakistan; \\ ${ }^{3}$ Medicinal and Aromatic Plants Research Institute, National Center for Research, Khartoum-11111, Sudan; ${ }^{4}$ Dr Panjwani Center for \\ Molecular Medicine \& Drug Research, International Center for Chemical \& Biological Sciences, University of Karachi, Karachi 75270, \\ Pakistan
}

Aim: To investigate the binding mode of synthesized adamantly derivatives inside of cholinesterase enzymes using molecular docking simulations.

Methods: A series of hybrid compounds containing adamantane and hydrazide moieties was designed and synthesized. Their inhibitory activities against acetylcholinesterase (AChE) and (butyrylcholinesterase) BChE were assessed in vitro. The binding mode of the compounds inside cholinesterase enzymes was investigated using Surflex-Dock package of Sybyl7.3 software.

Results: A total of 26 adamantyl derivatives were synthesized. Among them, adamantane-1-carboxylic acid hydrazide had an almost equal inhibitory activity towards both enzymes, whereas 10 other compounds exhibited moderate inhibitory activity against BChE. The molecular docking studies demonstrated that hydrophobic interactions between the compounds and their surrounding residues in the active site played predominant roles, while hydrophilic interactions were also found. When the compounds were docked inside each enzyme, they exhibited stronger interactions with BChE over AChE, possibly due to the larger active site of BChE. The binding affinities of the compounds for BChE and AChE estimated were in agreement with the experimental data.

Conclusion: The new adamantly derivatives selectively inhibit BChE with respect to AChE, thus making them good candidates for testing the hypothesis that BChE inhibitors would be more efficient and better tolerated than AChE inhibitors in the treatment of Alzheimer's disease.

Keywords: N-arylidene-1-adamantylcarbohydrazides; adamantane; hydrazide; cholinesterase inhibitor; Alzheimer's disease; acetylcholinesterase; butyrylcholinesterase; molecular docking simulation

Acta Pharmacologica Sinica (2015) 36: 879-886; doi: 10.1038/aps.2014.173; published online 4 May 2015

\section{Introduction}

Alzheimer's disease (AD), the most common form of dementia, is an increasing medical, social and public health concern ${ }^{[1]}$. $\mathrm{AD}$ is characterized by deficits in the cholinergic system and the deposition of $\beta$-amyloid $(A \beta)^{[2]}$. The cholinergic system has been targeted for the design of anti-Alzheimer's drugs. Cholinesterase inhibitors increase both the level and duration of action of acetylcholine. In addition, it has been demonstrated that both acetylcholinesterase and butyrylcholinesterase (BChE) play an important role in $\mathrm{A} \beta$-aggregation during

\footnotetext{
* To whom correspondence should be addressed.

E-mail zaheer.qasmi@iccs.edu (Zaheer UL-HAQ); alaboudi@ju.edu.jo (Amal AL-ABOUDI)

Received 2014-07-11 Accepted 2014-09-15
}

plaque formation $^{[3]}$. Many research papers published in the last decade have indicated that the structures of compounds that exhibit cholinesterase inhibitory activity are diverse ${ }^{[4,5]}$.

Adamantane derivatives are known to exhibit bioactivities that include antiviral ${ }^{[6]}$, antibacterial ${ }^{[7,8]}$, and anti-inflammatory $^{[9]}$ activities and inhibition of the central nervous system ${ }^{[10]}$ and $11 \beta-\mathrm{HSD}^{[11]}$.

Hydrazones are one of the most studied classes of compounds owing to their ease of synthesis, availability and diversity. Many hydrazones show inhibitory activities towards enzymes ${ }^{[12]}$. Various hydrazones have been shown to be strong antimicrobial, anti-inflammatory and antifungal agents $^{[13,14]}$. The antimicrobial agents, $(3 \alpha, 5 \beta, 7 \alpha, 12 \alpha)-3,7,12-$ trihydroxy-N-[(1E)-4-chlorophenylmethylene] cholan-24-hydrazide were introduced recently by our group ${ }^{[15]}$. The supe- 
riority of the designed compounds over the drugs Cefaclor and Cefixime, when tested against various bacterial strains, encouraged us to further investigate various hydrazone derivatives for new targets.

Inspired by the diverse bioactivities of molecules containing adamantane and hydrazide moieties, and as a continuation of our work on new cholinergic inhibitors ${ }^{[16]}$, we designed new hybrid compounds containing both moieties and tested their ability to inhibit acetylcholinesterase and butyrylcholinesterase.

Molecular docking is a powerful computational tool that can predict the interaction energy between two molecules (receptor and ligand) and determine which orientation of a ligand would form the lowest energy complex within the receptor's binding pocket ${ }^{[17-21]}$. In the Surflex docking methodology, Hammerhead's empirical scoring function is paired with a molecular similarity method to generate putative poses of ligand fragments. This process implements an incremental construction search approach and a new fragment assembly methodology; each is faster and more accurate. This new fragment assembly method is loosely related to the genetic algorithm approach, although it is a deterministic method.

Our docking results were in good agreement with experimental findings and demonstrated that the bulky adamantyl derivatives are more potent against $\mathrm{BChE}$ than $\mathrm{AChE}$ because $\mathrm{BChE}$ has a larger binding pocket. $\mathrm{BChE}$ also has wider substrate specificity and interacts with a broad range of inhibitors. As a result, the binding site of $\mathrm{BChE}$ accommodated adamantyl derivatives (1-26) relatively well, compared to AChE. Adamentyl derivatives synthesized recently by our group could be considered as lead compounds for developing AD treatments, as some selective BChE inhibitors have already been reported to increase acetylcholine levels and to reduce the formation of abnormal amyloid found in Alzheimer's disease ${ }^{[22,23]}$.

\section{Materials and methods} Materials and equipments

${ }^{1} \mathrm{H}$ NMR spectra were recorded on a Bruker Avance III-500 $\mathrm{MHz}$ spectrometer using tetramethyl silane as an internal standard. ${ }^{13} \mathrm{C}$ NMR spectra were recorded at $125 \mathrm{MHz}$ using a Bruker Avance III-500 MHz spectrometer and tetramethyl silane as an internal standard. Chemical shifts were reported as $\delta$-values in $\mathrm{ppm}$. The multiplicities of carbon atoms were determined from DEPT experiments. High resolution mass spectra (HRMS) were recorded in positive ion mode by electrospray ionization using a Bruker Daltonics Apex IV, 7.0 T Ultra Shield Plus. Samples were dissolved in chloroform, diluted in spray solution (methanol/water 1:1 $v / v+0.1 \%$ formic acid) and infused using a syringe pump with a flow rate of $2 \mu \mathrm{L} / \mathrm{min}$. External calibration was conducted using an arginine cluster in a mass range $m / z$ 175-871. For all HRMS data, mass error: $0.00-0.50 \mathrm{ppm}$. Melting points $(\mathrm{mp})$ were determined on an Electrothermal Melting point Apparatus and are reported uncorrected in ${ }^{\circ} \mathrm{C}$. Solvents used in this study were obtained from Scharlau, Fluka or Aldrich $<$. All reactions were monitored by thin layer chromatography (TLC) using Merck aluminum plates pre-coated with silica gel (PF254; $20 \times 20 \times 0.25$ $\mathrm{mm})$ and were detected by visualization of the plate under UV illumination $(\lambda=254$ or $365 \mathrm{~nm}$ ). Spots were also detected by spraying with anisaldehyde-sulfuric acid in ethanol, followed by heating to $140^{\circ} \mathrm{C}$.

\section{Cholinesterase inhibition assay}

Inhibition of $\mathrm{AChE}$ and $\mathrm{BChE}$ was measured in vitro using Ellman's spectrophotometric method ${ }^{[24]}$. Electric-eel AChE (Torpedo californica type VI-S, Sigma, EC 3.1.1.7), horse-serum BChE (EC 3.1.1.8), acetylthiocholine iodide (ATCh), butyrylthiocholine chloride (BTCh), 5,5-dithiobis[2-nitrobenzoic acid (DTNB) and Tacrine were purchased from Sigma (St Louis, MO, USA). Buffers and other chemicals were of analytical grade. Acetylthiocholine iodide and butyrylthiocholine chloride were used as substrates to assay $\mathrm{AChE}$ and $\mathrm{BChE}$, respectively. All inhibition studies were performed in 96-well microtiter-plates using a Bio Tek $\mu$ Quant ${ }^{\mathrm{TM}}$ microplate spectrophotometer.

\section{Methyl adamantane-1-carboxylate (2)}

Thionyl chloride $(15 \mathrm{~mL})$ was added drop wise with continuous stirring to a solution of adamantane-1-carboxylic acid $(5 \mathrm{~g}, 28 \mathrm{mmol})$ in dry methanol $(60 \mathrm{~mL})$ at $0^{\circ} \mathrm{C}$. The mixture was refluxed for $15 \mathrm{~h}$. Upon cooling, a crystalline solid precipitated and was collected via suction filtration and dried, yielding $4.8 \mathrm{~g}(96 \%)$ of methyl adamantane-1-carboxylate (mp $\left.37^{\circ} \mathrm{C}\right)$.

${ }^{1} \mathrm{H}$ NMR $\left(500 \mathrm{MHz}, \mathrm{CDCl}_{3}\right): \delta=1.72(\mathrm{~s}, 6 \mathrm{H}$, adamantane- $\mathrm{H})$, $1.89(\mathrm{~s}, 3 \mathrm{H}$, adamantane-H), $1.92(\mathrm{~s}, 6 \mathrm{H}$, adamantane- $\mathrm{H}), 3.68$ (s, $\left.\mathrm{CH}_{3}\right) \cdot{ }^{13} \mathrm{C}$ NMR $\left(\mathrm{CDCl}_{3}\right) \delta=27.9(\mathrm{C}-1, \mathrm{C}-5$, and $\mathrm{C}-7$ of adamantane), 36.5 (C-6, C-8, and C-9 of adamantane), 38.8 (C-2, $\mathrm{C}-4$, and $\mathrm{C}-10$ of adamantane), $40.7(\mathrm{C}-3), 51.52\left(\mathrm{CH}_{3}\right), 178.2$ (C-11). HRMS (ESI): calcd for $\mathrm{C}_{11} \mathrm{H}_{18} \mathrm{O}_{2}[\mathrm{M}+\mathrm{H}]^{+}: 217.11990$ found: 217.11960 .

\section{Adamantane-1-carboxylic acid hydrazide (3)}

A mixture of methyl adamantane-1-carboxylate $(4.8 \mathrm{~g}, 25$ $\mathrm{mmol}$ ) and $98 \%$ hydrazine hydrate $(12 \mathrm{~mL})$ in absolute ethanol $(18 \mathrm{~mL})$ was refluxed with stirring overnight. Upon the completion of the reaction (as checked by TLC), the reaction mixture was poured onto crushed ice to produce a white crystalline solid, which was filtered and washed with cold water to yield $4.4 \mathrm{~g}(93 \%)$ of adamantane-1-carboxylic acid hydrazide. $\left(\mathrm{mp}=150^{\circ} \mathrm{C}\right)$.

\section{$\mathrm{N}$-arylidene-1-adamantylcarbohydrazides (4a-w)}

The corresponding aromatic aldehyde $(1.1 \mathrm{mmol})$ was added to a solution of $1 \mathrm{mmol}$ of adamantane-1-carboxylic acid hydrazide in absolute ethanol containing 6 drops of glacial acetic acid. The mixture was refluxed for $4 \mathrm{~h}$, poured into cold water, and left standing for $1 \mathrm{~h}$ to complete precipitation. The precipitate formed was separated by filtration, washed with petroleum ether and air dried. Products were purified by silica gel column chromatography using a gradient of dichloromethane/ethylacetate solution as an eluent, followed by 
recrystallization from ethanol to yield pure compounds.

\section{Enzyme inhibition assay}

One hundred and thirty microliters of $0.1 \mu \mathrm{mol} / \mathrm{L}$ sodium phosphate buffer ( $\mathrm{pH} 8.0), 10 \mu \mathrm{L}$ of test compound solution with different concentrations, and $20 \mu \mathrm{L}$ of a $4 \times 10^{-7} \mathrm{mg} / \mathrm{mL}$ solution of acetylcholinesterase were mixed and incubated for $15 \mathrm{~min}$ at $25^{\circ} \mathrm{C}$. Twenty microliters of a solution of $8 \mathrm{mg}$ DTNB $3.25 \mathrm{mg}$ and $\mathrm{NaHCO}_{3}$ dissolved in $1.0 \mathrm{~mL}$ phosphate buffer $\mathrm{pH}=7.0$ was added to each well. The reaction was then initiated by addition of $20 \mu \mathrm{L}$ of $4.0 \mathrm{mg} / \mathrm{mL}$ acetylthiocholine iodide in deionized water. The hydrolysis of ATCh was monitored by measuring the formation of the yellow-colored 5-thio-2-nitrobenzoate anion at a wavelength of $412 \mathrm{~nm}$ for 5 min. The blank reading solution was used to justify nonenzymatic hydrolysis of substrate during the assay. The blank solution was prepared by replacing the enzyme solution with $20 \mu \mathrm{L}$ of buffer. An identical solution without inhibitor was processed following the same protocol. The same procedure was used for the butyrylcholinesterase inhibition assay, using a $0.02 \mathrm{mg} / \mathrm{mL}$ solution of BChE and $0.5 \mathrm{mg} / \mathrm{mL}$ of butyrylthiocholine chloride substrate concentration.

Test compounds were dissolved in ethanol while the control received the same volume of the solvent. All the reactions were performed in triplicate and the initial rate was measured as the rate of change in $\mathrm{OD} / \mathrm{min}$ (optical density/min) and used in subsequent calculations. $\mathrm{IC}_{50}$ values were determined by spectrophotometric measurement of the effect of increasing concentrations of test compounds on enzyme activity. The $\mathrm{IC}_{50}$ values were calculated using EZ-Fit Enzyme Kinetics Program.

\section{In-silico methodology}

Three dimensional (3D) molecular structures of twenty six adamantyl derivatives (1-26) along with tacrine as a standard were drawn by using the builder within MOE software. All constructed compounds were optimized and charges applied according to the MMFF94 protocol.

\section{Preparation of protein targets}

The X-ray crystal structures of both enzymes, human AChE (hAChE, PDB ID: 4EY5:2.30) and human BChE (hBChE, PDB ID: 2 WSL:2.0 ̊) were retrieved from Protein Data Bank (PDB), and whole structures were minimized after addition of polar hydrogen atoms.

Molecular docking studies were carried out to understand the binding mode of the synthesized adamantyl derivatives 1-26 inside each of the cholinesterase enzymes using the Surflex-Dock package of Sybyl7.3 ${ }^{[25-27]}$ software. Surflex is a fully automatic, flexible molecular docking algorithm that combines the scoring function from the Hammerhead docking system with a search engine that relies on a surface-based molecular similarity method as a means to rapidly generate suitable putative poses for molecular fragments.

Prior to docking, the 3D structures of inhibitors and recep- tors were prepared according to Surflex-Dock requirements. In Surflex-Dock, automatic active site detection of prepared proteins was conducted by "protomol" and used an empirically derived scoring function based on the binding affinity. In this study, standard parameters were used to estimate the binding affinities of new adamantyl derivatives characterized by the Surflex-Dock score (total score) expressed in $-\log _{10}\left(K_{\mathrm{d}}\right)$ units $^{[28]}$.

\section{Results and discussion Chemistry \\ Preparation of adamantane-1-carboxylic acid hydrazide (3)}

The synthesis of adamantane-1-carboxylic acid hydrazide (3) from adamantane-1-carboxylic acid (1) began with esterification of the acid by methanol in the presence of $\mathrm{SOCl}_{2}$ to give a white solid ester in quantitative yield (2). The ester (2) was recrystallized from ethanol and characterized by comparing the melting point to the published value. Direct esterification of (1) through acid catalysis was carried out by refluxing an ethanolic solution of the acid (1) in the presence of sulfuric $\operatorname{acid}^{[29]}$.

The hydrazide target compound was prepared by refluxing the ester with hydrazine hydrate in ethanol overnight. The reaction afforded the desired product (3) in 93\% yield (Scheme 1). The compound was identified by comparing its $\mathrm{mp}$ to the published value ${ }^{[30]}$. The ${ }^{1} \mathrm{H}-\mathrm{NMR}$ spectra of (3) confirmed substitution of the methoxy group by hydrazine, as it showed signals from the amine hydrogens and the absence of the signal from a methyl group.

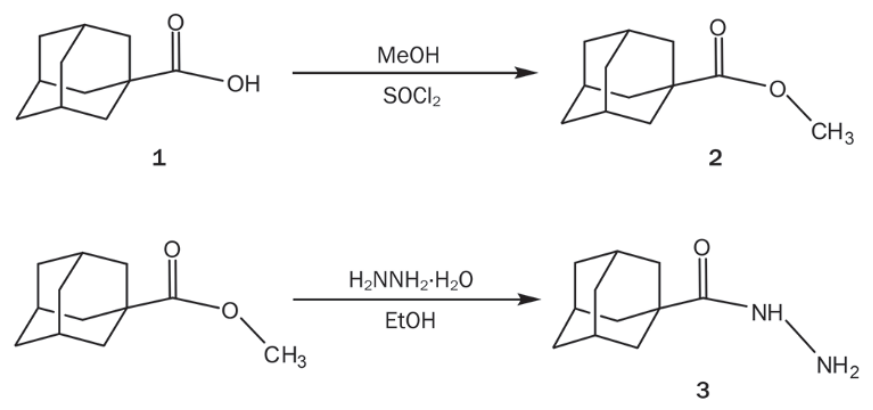

Scheme 1. Synthesis of adamantane-1-carboxylic acid hydrazide.

Preparation of $\mathrm{N}$-arylidene-1-adamantylcarbohydrazides (4a-w)

We used condensation of adamantane-1-carboxylic acid hydrazide (3) with different aldehydes as the core synthetic approach for generating this series, as shown in Scheme 2.

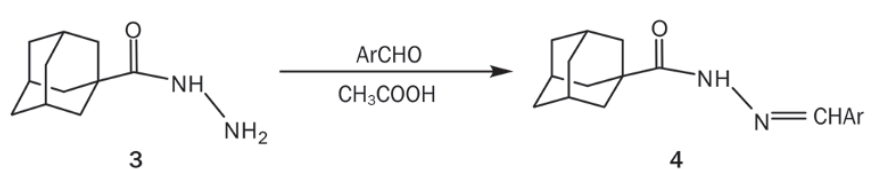

Scheme 2. N-arylidene-1-adamantylcarbohydrazide (4a-w) synthesis. 
Through this reaction, 23 different aromatic aldehydes were used to obtain the desired series of hydrazides. Of the synthesized compounds, 7 (4a-g) have been reported recently ${ }^{[31]}$ and the remaining $16(4 \mathbf{h}-\mathbf{w})$ were synthesized for the first time. All compounds were identified using physico-chemical techniques.

${ }^{1} \mathrm{H}$ NMR signals from the adamantane moiety appeared as a set of three broad singlets integrating for 6,6 and 2 protons in the range of $\delta 1.65-2.02 \mathrm{ppm}$. The aromatic protons appeared as expected in the range of $\delta 7.0-8.5 \mathrm{ppm}$, with multiplicities and coupling constants reflecting the substitution pattern of each compound. The ${ }^{1} \mathrm{H}-\mathrm{NMR}$ spectra of each of the synthesized compounds showed the signal from the methyne proton $(\mathrm{CH}=\mathrm{N})$ as a singlet in the range of $\delta 8.29-8.65 \mathrm{ppm}$, whereas that of the amide proton resonated as a singlet (sometime broad singlet) at $\delta 11.2-11.9 \mathrm{ppm}$. All spectra were in full agreement with the proposed structures. Compounds $4 \mathbf{h}, \mathbf{4 i}$, and $4 \mathbf{j}$ showed the protons of the hydroxy groups whereas the indole-containing compounds clearly showed the NH signal in the range of 10.5-13.0 ppm. The ${ }^{13} \mathrm{C}-\mathrm{NMR}$ spectral data of the hydrazones $(\mathbf{4 a - w})$ were in full agreement with the proposed structures. DEPT experiments were used to indicate the multiplicity of carbon atoms. The adamantane moiety showed four different peaks, two signals for $\mathrm{CH}_{2}$ carbons, one for $\mathrm{CH}$ carbons and one for a quaternary carbon atom. Aromatic carbons appeared in the range of $\delta 125-165 \mathrm{ppm}$. The $\mathrm{N}=\mathrm{CH}$ carbon signal appeared in the region of $\delta 138-148$ ppm, whereas that of the amide carbon $(-\mathrm{C}=\mathrm{ONH}-)$ resonated in the range of

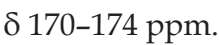

The methyl groups on the aromatic moiety resonated at the expected chemical shift of $\delta$ 20-25 ppm, whereas that of the methoxy group resonated in the range of $\delta 50-60 \mathrm{ppm}$. The hetero-aromatic compounds showed the anticipated chemical shifts for all of the carbon atoms compared to the known shifts reported in the literature.

High resolution mass spectra were in close agreement with the proposed formula of each compound.

\section{Cholinesterase inhibition studies}

The results (Table 1 ) indicated that adamantane derivatives did not exhibit inhibitory activity against AChE, except compound (3), which showed moderate activity with an $\mathrm{IC}_{50}$ of $17.8 \mu \mathrm{mol} / \mathrm{L}$. Compounds 1, 2, 3, 4d, 4h, 4j, 4k, 41, 4m, 4r, and $4 \mathrm{~s}$ were found to have moderate inhibitory activity against BChE, with $\mathrm{IC}_{50}$ values of 29.69, 8.65, 18.05, 10.01, 75.57, 10.75, $8.80,13.75,12.40,35.50$, and $29.19 \mu \mathrm{mol} / \mathrm{L}$, respectively. The remaining compounds were considered to be inactive because their $\mathrm{IC}_{50}$ values were more than $100 \mu \mathrm{mol} / \mathrm{L}$.

Differences in inhibitory activity within this series of compounds towards $\mathrm{AChE}$ and $\mathrm{BChE}$ can be attributed to differences in structure between these two enzymes. Nicolet et al ${ }^{[32]}$ indicated that, in spite of the similarities between the structures of the two enzymes, several aromatic residues lining the gorge of AChE are replaced by hydrophobic aliphatic residues in BChE. In addition, $\mathrm{Phe}^{288}$ and $\mathrm{Phe}^{290}$ in the acyl-binding pocket of AChE are replaced by $\mathrm{Leu}^{286}$ and $\mathrm{Val}^{288}$, respectively, in $\mathrm{BChE}$; these changes allow bulkier substrate moieties to bind more readily to $\mathrm{BChE}$ than to $\mathrm{AChE}$.

It has been reported that levels of $\mathrm{AChE}$ decline in the brain with the progress of $\mathrm{AD}$, which may limit the therapeutic value of AChE inhibitors. On the other hand, the activity of BChE increases in the brains of patients with $\mathrm{AD}^{[33]}$. Thus, the search for selective BChE inhibitors might be considered as an innovative approach for the treatment of $\mathrm{AD}^{[34]}$. Here, we report a new class of $\mathrm{N}$-arylidene-1-adamantylcarbohydrazides that may have promising therapeutic potential for AD.

The most active compound was methyl adamantane-1carboxylate (2), although its activity decreased upon conversion to the corresponding hydrazide (3). The activity of $\mathbf{4 k}$ was almost identical to that of (2). The importance of the N-phydroxyphenyl moiety for the activity of adamantylcarbohydrazides was confirmed by determining the inhibitory activity of 2,4-dihydroxyphenyl (41) and 2,3,4-trihydroxyphenyl-1adamantylcarboyhydrazides $(4 \mathrm{~m})$. The presence of more than one hydroxyl group did not enhance inhibitory activity.

Compound $\mathbf{4} \mathbf{j}$, which contains a methoxy group in place of a hydroxyl group at the p-position of the phenyl ring, showed almost the same $\mathrm{IC}_{50}$ as that of compound $4 \mathbf{k}$. This suggests that the oxygen atom at the p-position plays an important role in the activity of compounds $\mathbf{4 k}, \mathbf{4 1}, \mathbf{4 m}$, and $\mathbf{4 j}$. This is also evident when comparing the activity of the above mentioned four compounds with that of inactive $4 \mathrm{~h}$ (where phenyl is not substituted), $\mathbf{4 u}$ (in which the oxygen is at the $m$-position), or $4 \mathbf{i}$ (which has a methyl at the $p$-position). In the latter, the decrease in inhibitory activity might also be due to steric factors. Steric effects are indicated upon comparison of the activities of the $p$-bromo (4a), $p$-chloro (4b) and $p$-fluoro (4c) substituents of N-phenyl-1-adamantylcarbohydrazides, where the activity decreases as the size of the halogen increases. The difference in the reactivity between the two constitutional isomers of p-nitro (4d) and m-nitro (4e) can be attributed to both steric and electronic factors; the p-isomer showed moderate inhibitory activity whereas the $m$-isomer was inactive. Derivatives containing an indole ring (4r, $4 \mathbf{s}$, and $4 \mathbf{t})$ indicated the same trend, in which compounds $\mathbf{4 r}$ and $\mathbf{4 s}$ are considered as moderately active, whereas the larger $\mathbf{4 t}$ is not active.

\section{Computational studies}

Molecular docking studies were performed to provide a theory for the mechanism by which our adamantyl derivatives bind with the cholinesterase enzymes. To date, several ligand-cholinesterase complexes have been reported. Docking studies applied on experimentally resolved structures of BChE (PDB code: 2 WSL) and AChE (PDB code: 4EY5) were carried out using the Surflex-Dock package contained within the Sybyl7.3 program. In the literature, cholinesterase enzymes are divided into five major domains (residue numbers mentioned here relate to $\mathrm{BChE}$ ): 1) the peripheral active site (PAS) region (Asp68, Tyr330); 2) the catalytic active site (CAS) region (Ser196, His436, Glu325); 3) the acyl pocket region (Leu281, Trp229, Phe438); 4) the choline sub-site region (Trp80, Tyr126, Glu195); and 5) the oxyanion hole region (Gly114, Gly115, 
Table 1. Summary of the in vitro AChE and BChE activities of compounds 1-3, 4a-w, and tacrine.<smiles>[R]C=NNC(=O)C12CC3CC(CC(C3)C1)C2</smiles>

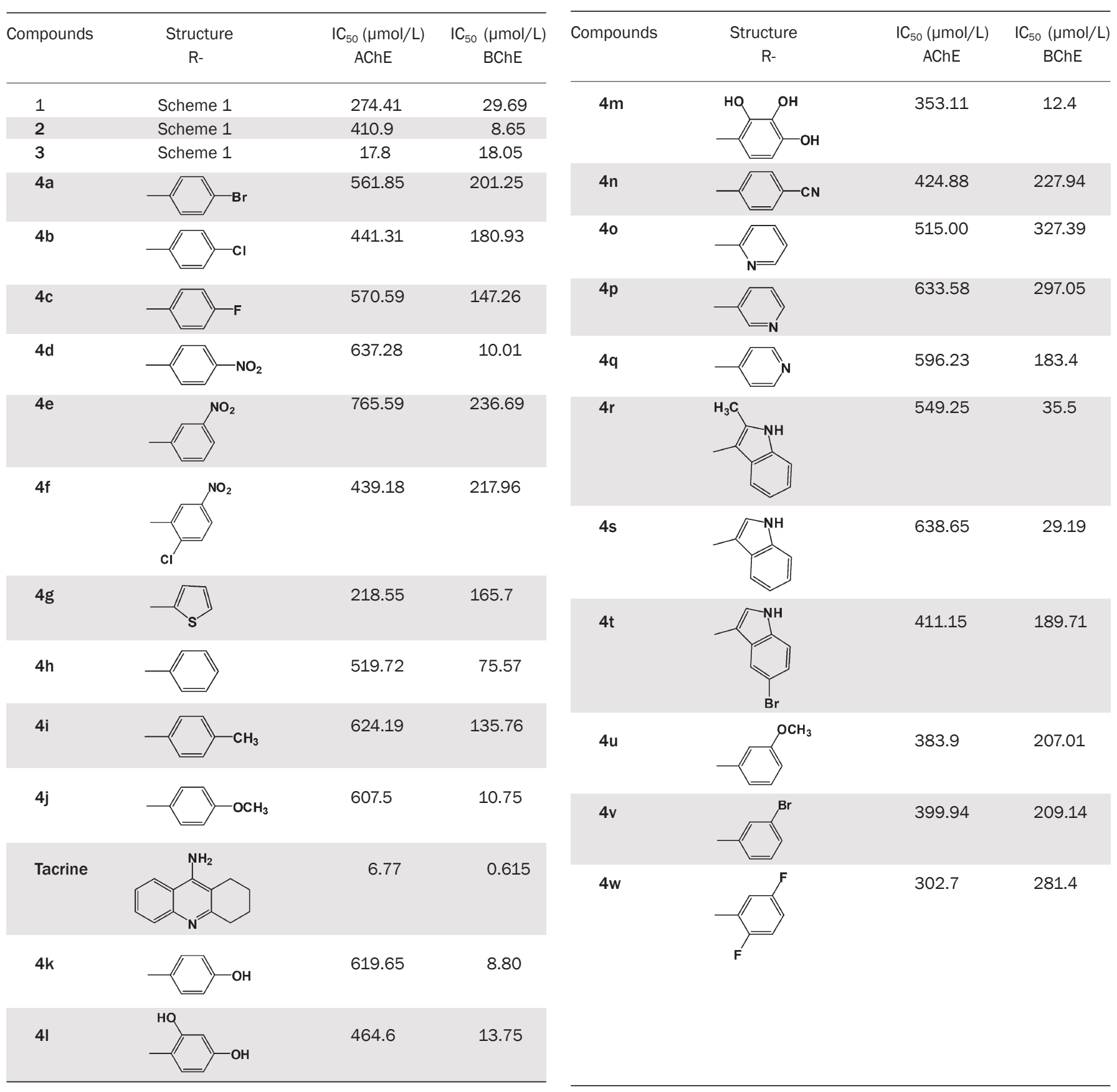

Ala197). The CAS residues of both proteins make a specific contribution to the inhibitors' specificity and selectivity.

Docking results demonstrated that FDA approved drugs such as tacrine (THA) bind more efficiently inside the binding cavity of $\mathrm{BChE}$ than $\mathrm{AChE}$, consistent with experimental results (Table 2). In the complex THA-BChE (Figure 1A), the THA moiety was bound to the CAS and cationic-n sites. Its structure is involved in $\Pi-\Pi$ bonding with the phenyl ring of $\operatorname{Trp} 80(2.94 \AA)$ by a parallel orientation and stabilized by a hydrogen bond with a CAS residue (His436) at a distance of $2.1 \AA$. However, in the THA-AChE complex, two п-п interactions with THA rings at distance of $3.29 \AA$ and $3.79 \AA$, respectively, were observed (Figure 1B).

On the basis of BChE experimental inhibition activities, 
Table 2. Binding affinities of tacrine with new adamantyl derivatives (1-26) against both of the cholinesterase enzymes (hAChE and hBChE).

\begin{tabular}{|c|c|c|c|}
\hline S № & $\begin{array}{l}\text { Compound } \\
\text { name }\end{array}$ & $\begin{array}{l}\text { Surflex-Dock } \\
\text { score (hBChE) }\end{array}$ & $\begin{array}{l}\text { Surflex-Dock } \\
\text { score (hAChE) }\end{array}$ \\
\hline 1 & 1 & 1.6 & 2.83 \\
\hline 2 & 2 & 1.22 & 3.63 \\
\hline 3 & 3 & 2.02 & 2.26 \\
\hline 4 & $4 a$ & 0.9 & 2.57 \\
\hline 5 & $4 b$ & 0.79 & 3.06 \\
\hline 6 & $4 c$ & 1.19 & 3.21 \\
\hline 7 & $4 d$ & 1.22 & 1.51 \\
\hline 8 & $4 e$ & 0.87 & 2.84 \\
\hline 9 & $4 f$ & 0.43 & 3.56 \\
\hline 10 & $4 g$ & 0.85 & 2.76 \\
\hline 11 & $4 h$ & 1.83 & 2.8 \\
\hline 12 & $4 i$ & 1.23 & 1.14 \\
\hline 13 & $4 j$ & 0.92 & 0.99 \\
\hline 14 & $4 k$ & 3.46 & 3.53 \\
\hline 15 & $4 I$ & 2.96 & 2.68 \\
\hline 16 & $4 m$ & 2.35 & 2.33 \\
\hline 17 & $4 n$ & 1.73 & 1.71 \\
\hline 18 & 40 & 1.51 & 3.3 \\
\hline 19 & $4 p$ & 1.49 & 2.5 \\
\hline 20 & $4 q$ & 0.92 & 3.45 \\
\hline 21 & $4 r$ & 1.61 & -0.2 \\
\hline 22 & $4 s$ & 2.88 & 2.91 \\
\hline 23 & $4 t$ & 2.67 & 3.23 \\
\hline 24 & $4 u$ & 0.88 & 3.75 \\
\hline 25 & $4 v$ & 1.02 & 2.94 \\
\hline 26 & $4 w$ & 1.46 & 2.65 \\
\hline 27 & Tacrine & 3.8 & 3.99 \\
\hline
\end{tabular}

twenty-six new compounds of adamantyl derivatives were categorized into three different groups for understanding the results of the docking study: active compounds $(0-79 \mu \mathrm{mol} / \mathrm{L})$, moderately active compounds $(135-250 \mu \mathrm{mol} / \mathrm{L})$ and inactive compounds ( $>250 \mu \mathrm{mol} / \mathrm{L})$. According to this classification, three different compounds were selected to explain the docking results against $\mathrm{BChE}$ and its results were correlated with experimental findings. Similar compounds were also used to understand the mechanism of interaction and inhibitory activities against $\mathrm{AChE}$ of adamantyl complexes.

\section{Active compounds $(0-79 \mu \mathrm{mol} / \mathrm{L})$}

Compound $1(29.69 \mu \mathrm{mol} / \mathrm{L})$ occupied the entire enzymatic CAS, mid-gorge and PAS regions, displaying several hydrophobic interactions. The hydroxyl $(\mathrm{OH})$ moiety also established a hydrogen bond $(1.96 \AA)$ with the carbonyl group of a CAS residue (His436, Figure 2). These interactions show that hydrophobic scaffolds exhibit an experimentally stronger inhibition compared to scaffolds that are hydrophilic in nature. These observations are also supported by comparison of compounds $4 \mathbf{k}, 4 \mathbf{l}$, and $4 \mathrm{~m}$, as a reduced inhibitory potency was observed as the number of hydroxyl groups on the phenyl ring were increased. These results are in agreement with experi-
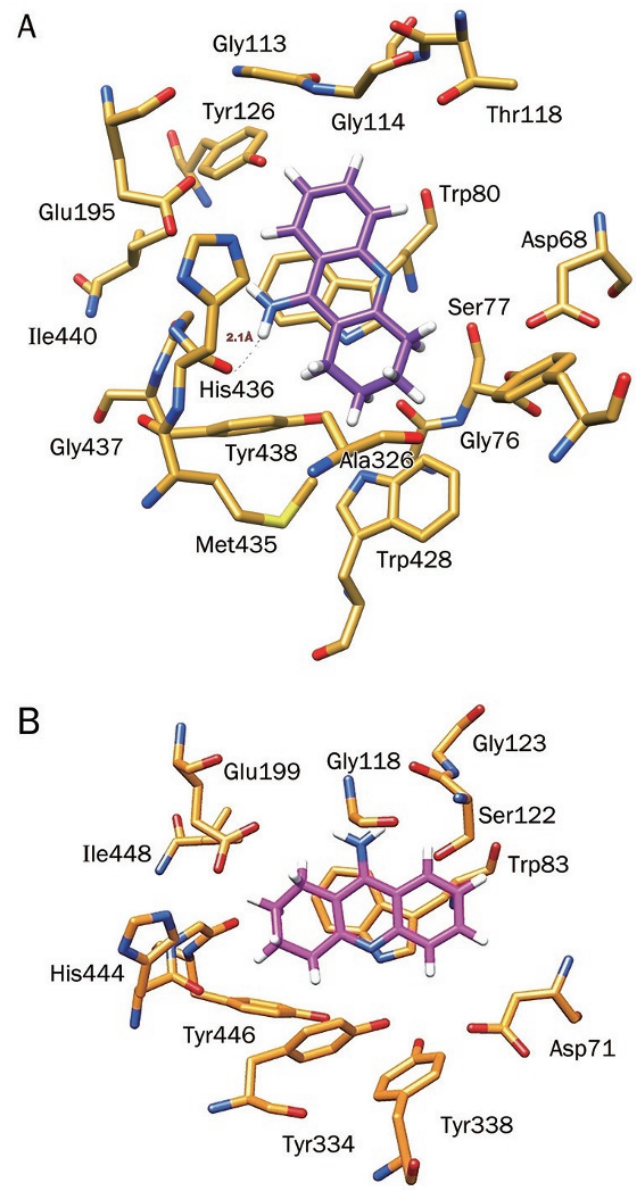

Figure 1. (A) Docked pose of the FDA approved drug tacrine as an active inhibitor inside the binding gorge of human butyrylcholinesterase ( $\mathrm{hBChE}$ ), (B) Docked pose of tacrine with human acetylcholinesterase (hAChE).

mental results. However, for $\mathrm{AChE}$, the important residues of the CAS, mid-gorge and PAS regions were not closely associated with the ligands. Owing to the relatively large distance, we could not establish significant interactions aside from week $\Pi-\mathrm{H}$ interactions between compound $1(274.4 \mu \mathrm{mol} / \mathrm{L})$ and the Trp86 residue (Figure 3).

\section{Least active compounds (135-250 $\mu \mathrm{mol} / \mathrm{L})$}

The molecular structure of $\mathbf{4 t}$ (189.71) reveals a large hydrophobic surface that is ideal for interacting with the hydrophobic lining of the $20 \AA$ deep gorge of BChE. Therefore, these residues can readily form hydrophobic interactions with the hydrophobic surface of $\mathbf{4 t}$, as well as interactions with the negatively charged, acidic residue Glu195 at a distance of $2.083 \AA$ (Figure 2) that contributed to the binding affinity of $\mathbf{4 t}$ to BChE. In the case of $\mathbf{4 a}, \mathbf{4 b}$, and $\mathbf{4 c}$, docking analysis specified that the size of the halogen had an effect on the inhibitory potency of these compounds against BChE. Eight hydrophobic active site residues of BChE were within $5 \AA$ of $4 \mathbf{t}$, whereas only six hydrophobic residues were contained within that radius of the binding pocket in AChE. This observation may account for the relatively stronger binding of $4 \mathbf{t}$ to $\mathrm{BChE}$ 
A
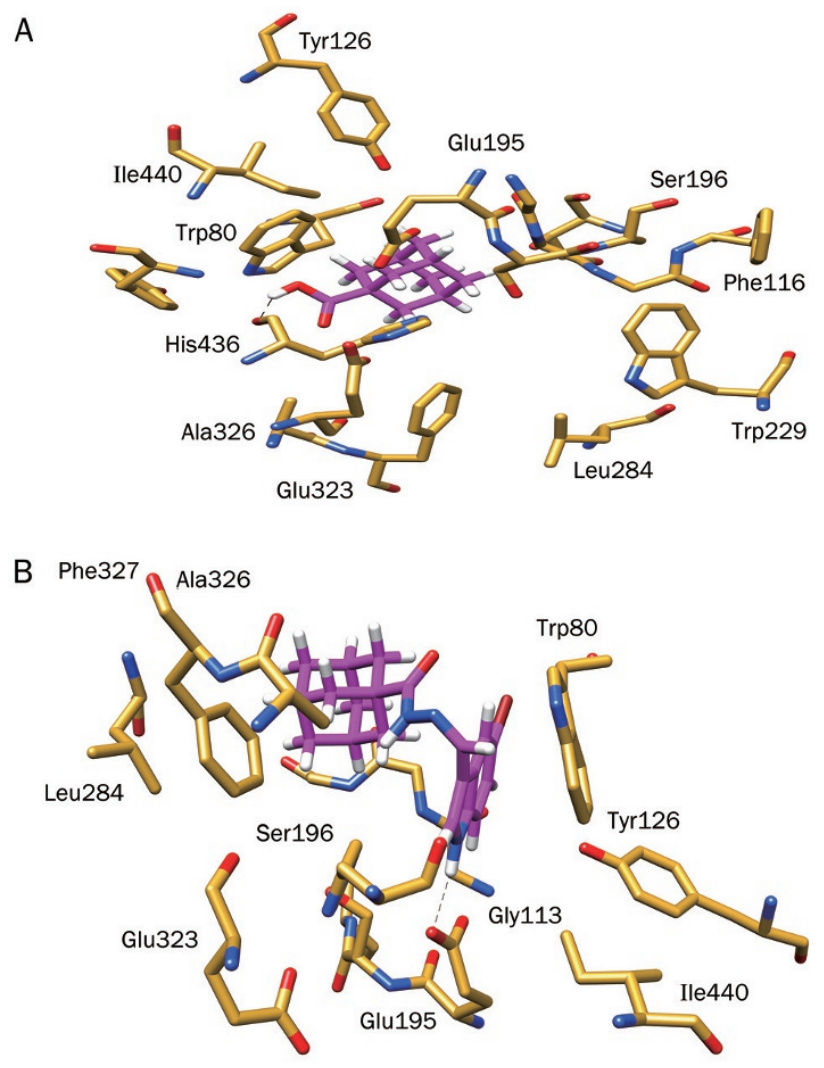

C

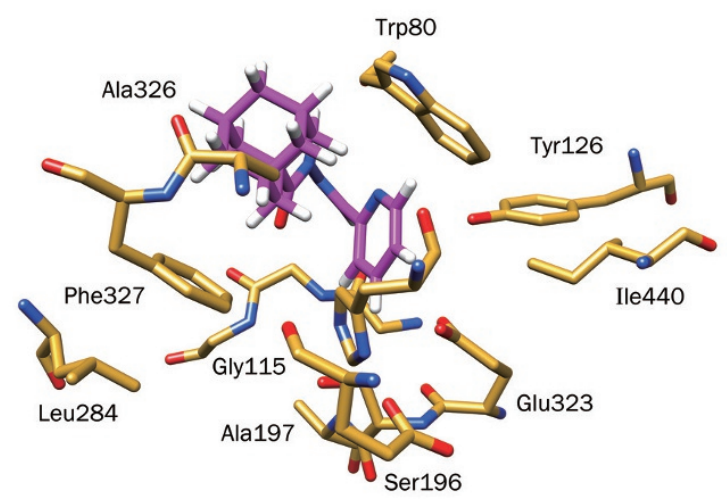

Figure 2. Putative binding mode of selected adamantyl derivatives according to their inhibition potency inside the binding gorge of human butyrylcholinesterase (hBChE): (A) active compound 1; (B) least active compound $\mathbf{4 t}$, and $(\mathrm{C})$ inactive compound $\mathbf{4 o}$.

than AChE (Figure 3). However, $4 \mathbf{t}$ also has the potential to form electrostatic interactions with active site residues of both enzymes, therefore, the inhibitory activity of $\mathbf{4 t}$ for each protein may differ primarily by hydrophobic interactions.

\section{Inactive (>250 $\mu \mathrm{mol} / \mathrm{L})$}

Compound $4 \mathbf{o}(327 \mu \mathrm{mol} / \mathrm{L})$ showed no-inhibitory potency towards $\mathrm{BChE}$ and has no H-bond interaction or other interactions to stabilize the complex system (Figure 2). Similarly, this compound was also inactive against AChE (Figure 3). Comparison of $4 \mathbf{o}$ with $\mathbf{4 t}$ reveals that the orientation of the ada-

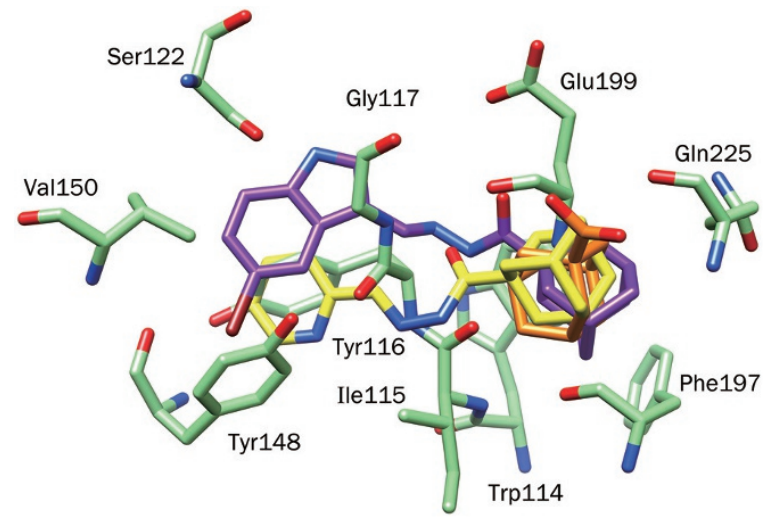

Figure 3. Representation of the top-scored docked poses of selected adamantyl derivatives to understand the binding pattern within the human acetylcholinesterase active site for compound $\mathbf{1}$ (orange), least active $\mathbf{4 t}$ (magenta) and inactive compound $\mathbf{4 0}$ (yellow).

mantyl moiety of $\mathbf{4 o}$ is relatively far away from the interactive hydrophobic residues because of the pyridine ring. This factor may be responsible for the lack of potency observed against both the cholinesterase enzymes.

\section{Conclusion}

Condensation of adamantane-1-carboxylic acid hydrazide with the corresponding aldehydes produced a series of $\mathrm{N}$-arylidene-1-adamantylcarbohydrazides in good yield. Screening the inhibitory activities of the compounds against each cholinesterase enzyme showed that all compounds were inactive towards AChE, but that several showed moderate activity towards BChE. Compound (3) shows an almost equal inhibitory activity towards both enzymes. The relatively low enzyme inhibition activities of the compounds may be due to steric effects and the large size of hydrazones. Additionally, theoretically estimated binding affinities are close to the range of experimental values. According to the binding modes, the hydrophobic interactions between adamantyl derivatives and their surrounding residues of the active site play predominant roles, while hydrophilic interactions were also found. When different adamantyl derivatives (1-26) were docked inside each cholinesterase enzyme (BChE and AChE), adamantyl compounds were selectively observed to inhibit $\mathrm{BChE}$ over $\mathrm{AChE}$, possibly due to the larger active site of $\mathrm{BChE}$.

\section{Acknowledgements}

The authors are grateful to the Deanship of Graduate Studies at the University of Jordan for financial support. Co-author (Zaheer UL-HAQ) is thankful to the Pakistan Science Foundation (PSF) for their financial support of computational studies.

\section{Author contribution}

Amal AL-ABOUDI has designed the research, wrote the paper and performed the in vitro enzyme inhibition studies; Raed A AL-QAWASMEH has designed the synthesis, supervised it and analyzed the spectral data; Alaa SHAHWAN has per- 
formed the synthesis; Uzma MAHMOOD has performed docking studies; Asaad KHALID has supervised the in vitro enzyme inhibition studies, and analyzed the results; Zaheer UL-HAQ has supervised the in sillico studies and analyzed the results.

\section{References}

1 Majnaric-Trtica L. Update on biological, social and economical aspect of Alzheimer's disease and other dementia. Period Biol 2009; 111: 381-8.

2 Laursen B, Mork A, Plath N, Kristiansen U, Bastlund JF. Impaired hippocampal acetylcholine release parallels spatial memory deficits in Tg2576 mice subjected to basal forebrain cholinergic degeneration. Brain Res 2014; 1543: 253-62.

3 Greig NH, Utsuki T, Yu Q, Zhu X, Holloway HW, Perry T, et al. A new therapeutic target in Alzheimer's disease treatment: attention to butyrylcholinesterase. Curr Med Res Opin 2001; 17: 159-65.

4 Loizzo MR, Tundis R, Menichini F, Menichini F. Natural products and their derivatives as cholinesterase inhibitors in the treatment of neurodegenerative disorders: an update. Curr Med Chem 2008; 15 : 1209-28.

5 Sener B, Orhan I. Exploring Turkish biodiversity: A rich source of chemical diversity for drug leads discovery. Pure Appl Chem 2011; 83: 1699-707.

6 Lekishvili N, Lekashvili O, Zurabishvili D, Fachulia Z, Giorgadze K. Synthesis and study of new adamantane-containing hydrazidehydrazone ligands and coordination compounds based on them. Oxid Commun 2012; 35: 457-75.

7 El-Emam AA, Alrashood KA, Al-Omar MA, Al-Tamimi AS. Synthesis and antimicrobial activity of $\mathrm{N}^{\prime}$-heteroarylidene-1-adamantylcarbohydrazides and ( \pm )-2-(1-adamantyl)-4-acetyl-5-5-(4-substituted phenyl-3-isoxazolyl)-1,3,4-oxadiazolines. Molecules 2012; 17: 347583.

8 Hassan GS, El-Emam AA, Gad LM, Barghash AM. Synthesis, antimicrobial and antiviral testing of some new 1-adamantyl analogues. Saudi Pharm J 2010; 18: 123-8.

9 Kadi AA, El-Brollosy NR, Al-Deeb OA, Habib EE, Ibrahim TM, El-Emam AA. Synthesis, antimicrobial, and anti-inflammatory activities of novel 2-(1-adamantyl)-5-substituted-1,3,4-oxadiazoles and 2-(1-adamantylamino)-5-substituted-1,3,4-thiadiazoles. Eur J Med Chem 2007; 42: 235-42.

10 Makovec F, Artusi R, Zanzola S, Rovati LC. Adamantane derivatives with neuroprotective, antidepressant and anti-ischaemic activities. U S Pat 2006; 7145037 B2.

11 Yeh VSC, Patel JR, Yong H, Kurukulasuriya R, Fung S, Monzon K, et al. Synthesis and biological evaluation of heterocycle containing adamantane 11B-HSD1 inhibitors. Bioorg Med Chem Lett 2006; 16 : 5414-19.

12 Chohan ZH, Pervez H, Khan KM, Supuran CT. Organometallic-based antibacterial and antifungal compounds: transition metal complexes of 1,1'-diacetylferrocene-derived thiocarbohydrazone, carbohydrazone, thiosemicarbazone and semicarbazone. J Enzyme Inhib Med Chem 2005; 20: 81-9.

13 Rollas S, Gulerman N, Erdeniz H. Synthesis and antimicrobial activity of some new hydrazones of 4-fluorobenzoic acid hydrazide and 3-acetyl-2,5-disubstituted-1,3,4-oxadiazolines. Farmaco 2002; 57: 171-4.

14 Bedia KK, Elcin O, Seda U, Fatma K, Nathaly S, Sevim R, et al. Synthesis and characterization of novel hydrazide-hydrazones and the study of their structure-antituberculosis activity. Eur J Med Chem 2006; 41: 1253-61.
15 Rasras AJ, Al-Tel TH, Al-Aboudi A, Al-Qawasmeh RA. Synthesis and antimicrobial activity of cholic acid hydrazone analogues. Eur J Med Chem 2010; 45: 2307-13.

16 Al-Aboudi A, Odeh H, Khalid A, Naz Q, Choudhary MI. Butyrylcholinesterase inhibitory activity of testosterone and some of its metabolites. J Enzyme Inhib Med Chem 2009; 24: 553-8.

17 Huang N, Shoichet BK, Irwin JJ. Benchmarking sets for molecular docking. J Med chem 2006; 49: 6789-801.

18 Erickson J A, Jalaie M, Robertson DH, Lewis RA, Vieth M. Lessons in molecular recognition: the effects of ligand and protein flexibility on molecular docking accuracy. J Med Chem 2004; 47: 45-55.

19 Clark M, Meshkat S, Talbot GT, Carnevali P, Wiseman JS. Fragmentbased computation of binding free energies by systematic sampling. J Chem Inf Model 2009; 49: 1901-13.

20 Jain AN. Bias, reporting, and sharing: computational evaluations of docking methods. J Comput Aided Mol Des 2008; 22: 201-12.

21 Kellenberger E, Rodrigo J, Muller P, Rognan D. Comparative evaluation of eight docking tools for docking and virtual screening accuracy. Proteins 2004; 57: 225-42.

22 Chiou SY, Huang CF, Hwang MT, Lin G. Comparison of active sites of butyrylcholinesterase and acetylcholinesterase based on inhibition by geometric isomers of benzene-di-N-substituted carbamates. J Biochem Mol Toxicol 2009; 23: 303-8.

23 Sekutor M, Mlinaric-Majerski K, Hrenar T, Tomic S, Primozic I. Adamantane-substituted guanylhydrazones: novel inhibitors of butyrylcholinesterase. Bioorg Chem 2012; 41-42: 28-34.

24 Ellman GL, Courtney KD, Featherstone RM. A new and rapid colorimetric determination of acetylcholinesterase activity. Biochem Pharmacol 1961; 7: 88-95.

25 Spitzer R, Jain AN. Surflex-Dock: Docking benchmarks and real-world application J Comput Aided Mol Des 2012; 26: 687-99.

26 Jain AN. Surflex-Dock 2.1: robust performance from ligand energetic modeling, ring flexibility, and knowledge-based search. J Comput Aided Mol Des 2007; 21: 281-306.

27 Miteva MA, Lee WH, Montes MO, Villoutreix BO. Fast structure-based virtual ligand screening combining FRED, DOCK, and Surflex. J Med Chem 2005; 48: 6012-22.

28 Jain AN. Surflex: fully automatic flexible molecular docking using a molecular similarity-based search engine. J Med Chem 2003; 46: 499-511.

29 Pacchioni G, Clotet A, Marchand AP, Dongxia X, Bott SG. Synthesis of (Z)- and (E)-1,2-di(1-adamantyl)ethene and their respective reactions with dichlorocarbene. Tetrahedron 1996: 52: 825-32.

30 Bormasheva KM, Nechaeva ON, Moiseev IK. Reactions of adamantylsubstituted keto esters with hydrazine and phenylhydrazine. Russ J Org Chem 2008; 44: 1760-4.

31 Al-Omar MA, Al-Abdullah ES, Shehata IA, Habib EE, Ibrahim TM, ElEmam AA. Synthesis, antimicrobial, and anti-inflammatory activities of novel 5-(1-adamantyl)-4-arylideneamino-3-mercapto-1,2,4-triazoles and related derivatives. Molecules 2010; 15: 2526-50.

32 Nicolet Y, Lockridge O, Masson P, Fontecilla-Camps JC, Nachon F. Crystal structure of human butyrylcholinesterase and of its complexes with substrate and products. J Biol Chem 2003; 278: 41141-7.

33 Arendt T, Brückner MK, Lange M, Bigl V. Changes in acetylcholinesterase and butyrylcholinesterase in Alzheimer's disease resemble embryonic development - a study of molecular forms. Neurochem Int 1992; 21: 381-96.

34 Kamal MA, Qu X, Yu QS, Tweedie D, Holloway HW, Li Y, et al. Tetrahydrofurobenzofuran cymserine, a potent butyrylcholinesterase inhibitor and experimental Alzheimer drug candidate, enzyme kinetic analysis. J Neural Transm 2008; 115: 889-98. 